\title{
Electron-state control of carbon nanotubes by space and encapsulated fullerenes
}

\author{
Susumu Okada, ${ }^{1,2}$ Minoru Otani ${ }^{1}$ and Atsushi Oshiyama ${ }^{1,2}$ \\ ${ }^{1}$ Institute of Physics, University of Tsukuba, Tennodai, Tsukuba 305-8571, Japan \\ ${ }^{2}$ Center for Computational Physics, University of Tsukuba, Tennodai, Tsukuba 305-8577, Japan
}

(Received 5 December 2002; revised manuscript received 4 March 2003; published 15 May 2003)

\begin{abstract}
We report total-energy electronic structure calculations that provide energetics of encapsulation of various fullerenes in carbon nanotubes and electronic structures of resulting carbon peapods. We find that the electron states of the peapods depend on the space in the nanotubes and that they reflect electron states of the encapsulated fullerenes. The deep energy position of the lowest unoccupied state of fullerenes as well as hybridization between $\pi$ states of the fullerenes and the nearly free-electron states of the nanotubes causes a multicarrier character in the peapods.
\end{abstract}

DOI: 10.1103/PhysRevB.67.205411

PACS number(s): 73.20.At, 73.22.-f, 71.20.Tx

Tubular structures of hexagonally bonded carbon atoms (carbon nanotubes) discovered in 1991 (Ref. 1) are known to exhibit a rich variety in their electronic structures: ${ }^{2,3}$ For instance, the nanotube with chiral index $(n, m)$ is a metal when $|n-m|$ is a multiple of three, whereas they are semiconducting otherwise. The peculiar electronic property is due to an additional boundary condition imposed on electron states of a graphite sheet, which is rolled into each nanotube. On the other hand, the nanotube is fascinating as a host material to induce various kinds of complexes by containing atoms or molecules inside or outside. ${ }^{4-7}$ Carbon nanotubes intercalated with alkaline or hydrogen atoms have been synthesized and are now attracting much attention not only in their interesting physical properties but also in industrial applications. Recently high resolution transmission electron microscope (TEM) images clearly showed the encapsulation of $\mathrm{C}_{60} \mathrm{~S}$ in nanotubes. ${ }^{8-12}$ In addition to $\mathrm{C}_{60}$, larger fullerenes, ${ }^{13} \mathrm{e}$ e.g., $\mathrm{C}_{70}, \mathrm{C}_{78}, \mathrm{C}_{80}$, and $\mathrm{C}_{84}$, and metallofullerenes, ${ }^{14}$ e.g., $\mathrm{Gd} @ \mathrm{C}_{82}$ and $\mathrm{Sc}_{2} \mathrm{C}_{84}$, were also found to be encapsulated in nanotubes. These unusual nanometer-scale carbon networks are now called "carbon peapods" of which structures are characterized by an interesting combination of one- and zerodimensional constituent units, i.e., carbon nanotubes and fullerenes. The peapods are thus regarded as hierarchical solids with mixed dimensionality.

The spaces inside the nanotubes or between them are known to be utilized for a container of atoms and molecules. Yet the space not only works as a container but also controls electron states in those materials. The space induces the peculiar delocalized electron states, which is located below a vacuum level and distributed not near atomic sites but in the space, and is of nearly free electron (NFE) character. ${ }^{15,16}$ The NFE states play crucial roles for the intercalation of atoms and control the electron states near the Fermi level $\left(E_{F}\right)$ of nanotubes. ${ }^{15,17}$ In graphite there is a counterpart named as an interlayer state, and it is important to consider excitation spectrum. ${ }^{18-20}$ In the peapods, owing to hybridization between $\pi$ states of $\mathrm{C}_{60}$ and the NFE states of the nanotube, a $\mathrm{C}_{60} @(10,10)$ peapod was found to be a metal with multicarriers each of which distributes either along the nanotube or on the $\mathrm{C}_{60}$ chain. $^{21}$ The results have indicated that the encapsulation of fullerenes within the nanotubes controls the electron states near $E_{F}$ of resultant materials.
We here report total-energy calculations performed for one-dimensional chains of $\mathrm{C}_{60}$, which are encapsulated in armchair nanotubes. We take $(n, n)$ nanotubes $(n=9,10,11$, 12 , and 13) and clarify energetics and electronic structures of the $\mathrm{C}_{60}$-peapods. Based on the results, we give the quantitative analysis for the energy shift of $\pi$ electron states of the $\mathrm{C}_{60}$ encapsulated in the $(n, n)$ armchair nanotubes, and clarify the correlation between the energy shift and the interunit spacing. Furthermore, we also study the electronic structures of the peapods consisting of the fullerenes $\left(\mathrm{C}_{70}\right.$ and $\mathrm{C}_{78}$ ), since the $\mathrm{C}_{70}$ peapod and $\mathrm{C}_{78}$ peapod are indeed synthesized recently. ${ }^{13}$ We have found that the electron states near $E_{F}$ of the peapods depend crucially on the interwall spacing between fullerenes and nanotubes, and that the size and shapes of the encapsulated fullerenes add important variety to the electronic structures of the peapods.

All calculations have been performed using the localdensity approximation (LDA) in the density-functional theory. ${ }^{22,23}$ For the exchange-correlation energy among electrons, we use a functional form ${ }^{24}$ fitted to the Monte Carlo results for the homogeneous electron gas. ${ }^{25}$ Normconserving pseudopotentials generated by using the Troullier-Martins scheme are adopted to describe the electron-ion interaction. ${ }^{26,27}$ In constructing the pseudopotentials, core radii adopted for $\mathrm{C} 2 s$ and $2 p$ states are both 1.5 Bohrs. The valence wave functions are expanded by the plane-wave basis set with a cutoff energy of $50 \mathrm{Ry}$, which is known to give enough convergence of total energy to discuss the relative stability of various carbon phases. ${ }^{26}$ We carry out the calculation for $(n, n)$ nanotubes $(10 \leqslant n \leqslant 13)$ in this paper. We adopt a supercell model in which a peapod is placed with its nanotube wall being separated by $6.5 \AA$ from another wall of an adjacent peapod. The conjugate-gradient minimization scheme is utilized both for the electronic-structure calculation and for the geometry optimization. ${ }^{28}$ In the geometry optimization, we impose a commensurability condition between the one-dimensional periodicity of the nanotube and that of the chain of $\mathrm{C}_{60}$ s. Consequently, the lattice parameter $c$ becomes $9.824 \AA$ along the tube direction which corresponds to the quadruple of the periodicity of the armchair nanotube. Integration over one-dimensional Brillouin zone is carried out using the two $k$ points. 


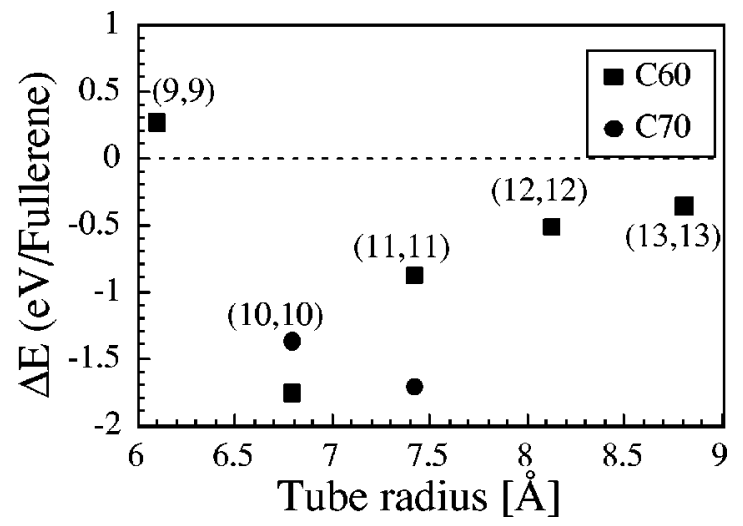

FIG. 1. Reaction energies $\Delta E$ (see text) per $\mathrm{C}_{60}$ in the encapsulation reaction for the $(9,9),(10,10),(11,11),(12,12)$, and $(13,13)$ armchair nanotubes (solid squares) Ref. 29. The value for $\mathrm{C}_{60} @(9,9)$ is from Ref. 21 . The reaction energy for $\mathrm{C}_{60} @(13,13)$ is computed with the 40-Ry cutoff energy for the basis set. Reaction energy $\Delta E$ per $\mathrm{C}_{70}$ in the encapsulation for the $(10,10)$ and $(11,11)$ nanotubes (solid circles).

We begin with stability of the $\mathrm{C}_{60}$ peapods with various tube radii. The stability is evaluated by calculating the energy difference in the following reaction:

$$
(n, n) \text { tube }+\mathrm{C}_{60} \rightarrow \mathrm{C}_{60} @(n, n)-\Delta E .
$$

Figure 1 shows the energy $\Delta E$ of the $\mathrm{C}_{60}$-peapods as a function of the tube radius. It is found that the reaction is exothermic for all tubes calculated here except $(9,9)$. The reaction for the $(10,10)$ tube is found to be the most favorable among the tubes studied here; the energy $\Delta E$ is $-1.73 \mathrm{eV}$ per $\mathrm{C}_{60}$. The optimized value of interwall spacing between the $\mathrm{C}_{60}$ and the $(10,10)$ nanotubes is $3.31 \AA$, which is close to the interlayer spacing of the graphite. On the other hand, the energy $\Delta E$ gradually approaches zero by increasing the tube radius. This is due to that $\mathrm{C}_{60} \mathrm{~s}$ are located at the center of the nanotubes. However, it is expected that $\mathrm{C}_{60} \mathrm{~s}$ may be located near the wall of the nanotube with an appropriate wall- $\mathrm{C}_{60}$ distance of about $3 \AA$ for the thick nanotubes. Indeed, the total-energy calculations clearly show that the optimum position of $\mathrm{C}_{60}$ in the $(12,12)$ nanotube is dislodged from the center of the nanotube by $1.6 \AA$ (Fig. 2). In this case, the interwall distance between $\mathrm{C}_{60}$ and the nanotube is about 3.1

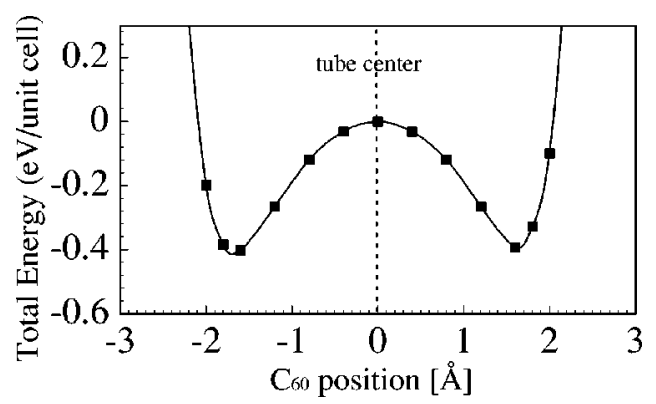

FIG. 2. Total energies of $\mathrm{C}_{60} @(12,12)$ per unit cell for the various radial positions of $\mathrm{C}_{60}$ in the $(12,12)$ nanotube. The energies are measured from that of $\mathrm{C}_{60} @(12,12)$ in which $\mathrm{C}_{60} \mathrm{~s}$ are located at the center of the nanotube.

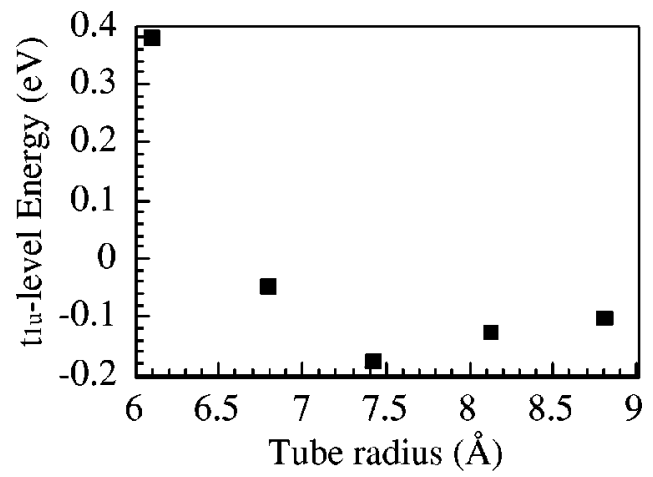

FIG. 3. The $t_{1 u}$ energy level (Kohn-Sham level) as a function of the tube radius in $\mathrm{C}_{60} @(n, n)$ in which $\mathrm{C}_{60} \mathrm{~s}$ are located at the center of the nanotube. In the optimum position, the $t_{1 u}$ state is slightly shifted upward compared to the case in which $\mathrm{C}_{60}$ is at the center. The energy levels are measured from $E_{F}$ of the empty $(n, n)$ tubes.

$\AA$ and the reaction energy $\Delta E$ is $-0.91 \mathrm{eV}$ per $\mathrm{C}_{60}$. The results indicate that $\mathrm{C}_{60}$ encapsulated in thick nanotubes are located at about $6.5 \AA$ off from the wall of nanotube to keep the preferable interwall distance of about $3 \AA$. Furthermore, the energy $\Delta E$ approaches not zero but a finite value of about $-0.4 \mathrm{eV}$. Recent TEM images indeed showed that $\mathrm{C}_{60} \mathrm{~s}$ are located near the wall of nanotubes rather than at the center of the nanotube. ${ }^{30}$

$\mathrm{C}_{60} @(10,10)$ has been reported ${ }^{21}$ to possess an interesting electronic structure around the Fermi level as explained below. In an isolated $\mathrm{C}_{60}$, there are the fivefold degenerate highest occupied $h_{u}$ state and the threefold degenerate lowest unoccupied $t_{1 u}$ state. In the $\mathrm{C}_{60}$ chain, both $h_{u}$ and $t_{1 u}$ show the dispersion of $\sim 0.6 \mathrm{eV}$ and become valence and conduction bands, respectively. The energy gap remains finite and is about $1 \mathrm{eV}$. As for the $(n, n)$ nanotube, there are two energy bands near Fermi energy, which cross $\varepsilon_{F}$ at $k \simeq 2 \pi / 3 c$ of Brillouin zone. ${ }^{2}$ Therefore, it may be expected in $\mathrm{C}_{60} @(n, n)$ that the two bands originated from the nanotube cross $\varepsilon_{\mathrm{F}}$ and are located in the gap of $\mathrm{C}_{60}$. However, in $\mathrm{C}_{60} @(10,10)$, four bands cross the Fermi level and $\mathrm{C}_{60} @(10,10)$ is a metal with multicarriers each of which is distributed on either nanotubes or the $\mathrm{C}_{60}$ chain.

Here we give quantitative studies on that unusual feature of $\mathrm{C}_{60} @(n, n)$. Figure 3 shows the relative energy of the bottom of the $t_{1 u}$-like state of $\mathrm{C}_{60} @(n, n)$ with respect to the Fermi level energy of the empty $(n, n)$ nanotubes. It is found that the energy shift of the $t_{1 u}$-like state of peapods depends on the tube radius. For the $(9,9)$ tube, the interwall spacing is insufficient to allow the $t_{1 u}$ state of $\mathrm{C}_{60}$ to distribute in the space so that $t_{1 u}$-like state shifts upwards and $\mathrm{C}_{60} @(9,9)$ does not exhibit the multicarrier character. On the other hand, the $t_{1 u}$-like state of $\mathrm{C}_{60} @(n, n)$ for which the encapsulation reaction is exothermic emerges below $E_{F}$ of the $(n, n)$ nanotubes so that the peapods exhibit the multicarrier character. The shift for $(11,11)$ is larger than those for other tubes. The shift gradually approaches zero with increasing the tube radius. The result is a strong evidence that the hybridization between the NFE states and the $\pi$ states indeed plays an important role to determine the electron states near $E_{F}$ of the peapods. 
(a) C60@(11,11)

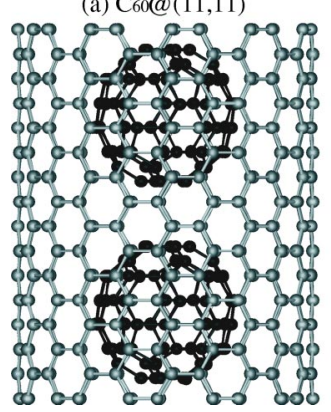

(b) C70@(11,11)

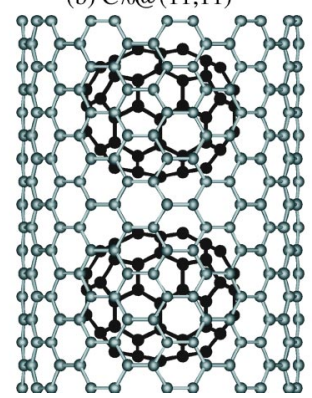

(c) 78 @(11,11)

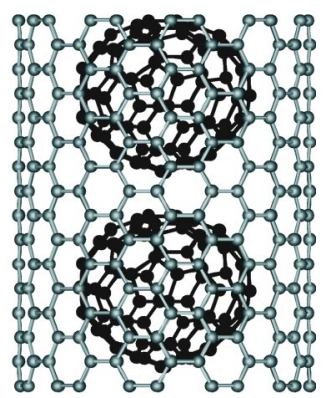

FIG. 4. Geometric structures of (a) $\mathrm{C}_{60} @(11,11), \quad$ (b) $\quad \mathrm{C}_{70} @(11,11), \quad$ and $\quad$ (c)
The multicarrier characters of the $\mathrm{C}_{60}$ peapods imply electron transfer from the nanotube to $\mathrm{C}_{60}$. Our previous work clearly shows an interesting charge redistribution on the encapsulation process of $\mathrm{C}_{60}$ inside the $(10,10)$ nanotube. ${ }^{21}$ The distribution of accumulated electrons corresponds to that of the NFE state of the nanotubes and implies that the hybridization indeed takes place. It is important to give a quantitative evaluation for the charge transfer on the system. We thus calculate the core level shift $(\Delta)$ of the $1 s$ state of a carbon atom in the nanotubes. The shift corresponds to the total energy difference between the neutral and the charged systems:

$$
\begin{aligned}
\Delta= & E_{\text {hole }}\left(\mathrm{C}_{60} @ \text { tube }\right)-E\left(\mathrm{C}_{60} @ \text { tube }\right) \\
& -\left[E_{\text {hole }}(\text { tube })-E(\text { tube })\right],
\end{aligned}
$$

where $E_{\text {hole }}\left(\mathrm{C}_{60} @\right.$ tube $), E\left(\mathrm{C}_{60} @\right.$ tube $), E_{\text {hole }}($ tube $)$, and $E$ (tube) are total energies of the peapod with an $1 s$ hole, the neutral peapod, the tube with an $1 s$ hole, and the neutral tube, respectively. In the total energy calculations with the $1 s$ hole, we first generate pseudopotentials with the $1 s$ hole, place them at an atomic site in either peapods or nanotubes, and perform the total-energy calculations. The calculated values of $\Delta$ are $7 \mathrm{meV}$ and $14 \mathrm{meV}$ for $\mathrm{C}_{60} @(10,10)$ and $\mathrm{C}_{60} @(11,11)$, respectively.

As indicated above, the electron states of the peapods near $E_{F}$ are tunable by controlling the radius of the nanotubes. On the other hand, it is also of interest how the electronic structure of the peapods depends on the encapsulated fullerenes. Here, we calculate the electronic structures of the $(11,11)$ nanotube containing $\mathrm{C}_{70}$ and $\mathrm{C}_{78}\left(C_{2 v}^{\prime}\right)$ fullerenes for which geometries have been already identified. ${ }^{31}$ The geometries of the $C_{70}$ peapod and the $C_{78}$ peapod are shown in Fig. 4. For the $\mathrm{C}_{70}$ peapods, we calculate the reaction energy $\Delta E$ per $\mathrm{C}_{70}$ in the formation of encapsulated structures for the $(10,10)$ and $(11,11)$ nanotubes (Fig. 1). In both cases, the reactions are found to be exothermic and the calculated $\Delta E$ are $-1.36 \mathrm{eV}$ and $-1.71 \mathrm{eV}$ for the $(10,10)$ and $(11,11)$ nanotubes, respectively. Figure 5 shows the electronic structures of $\mathrm{C}_{60} @(11,11), \mathrm{C}_{70} @(11,11)$, and $\mathrm{C}_{78} @(11,11)$. In an isolated $\mathrm{C}_{70}$ molecule, there are the triply bunched highest occupied (HO) states and the triply bunched lowest unoccupied (LU) states. In the $\mathrm{C}_{70}$ chain, both $\mathrm{HO}$ and LU states show the dispersion of about $0.6 \mathrm{eV}$ and become valence and conduction bands, respectively, with the band gap of about $1.6 \mathrm{eV}$. For $\mathrm{C}_{70} @(11,11)$, it is found that only two linear dispersion bands originated from the $\pi$ and $\pi^{*}$ states of the nanotube emerge at $E_{F}$ and the LU band of $\mathrm{C}_{70}$ is located above $E_{F}$ by $0.04 \mathrm{meV}$. Thus, transport characteristics of the system are different from that of the $\mathrm{C}_{60}$ peapods, and the carriers are distributed only on the nanotube. In an isolated $\mathrm{C}_{70}$ molecule, our LDA calculations show that the LU state is higher in energy by $0.25 \mathrm{eV}$ than the $t_{1 u}$ state of a $\mathrm{C}_{60}$ molecule. The higher LU state of the $\mathrm{C}_{70}$ does not cause a multicarrier character even though there is a sufficient downward shift of the $\pi$ states due to the $\pi$-NFE hybridization. This indicates that the electronic structure of the peapods depends on the electron states of the encapsulated fullerenes. Further, the peapods consisting of fullerenes whose LU state is lower than the $t_{1 u}$ state of $\mathrm{C}_{60}$ are expected to exhibit the multicarrier character.

We thus consider a major isomer of the $\mathrm{C}_{78}$ fullerene, i.e., $C_{2 v}^{\prime}$ isomer, ${ }^{31}$ of which the LU state is lower by $0.1 \mathrm{eV}$ than
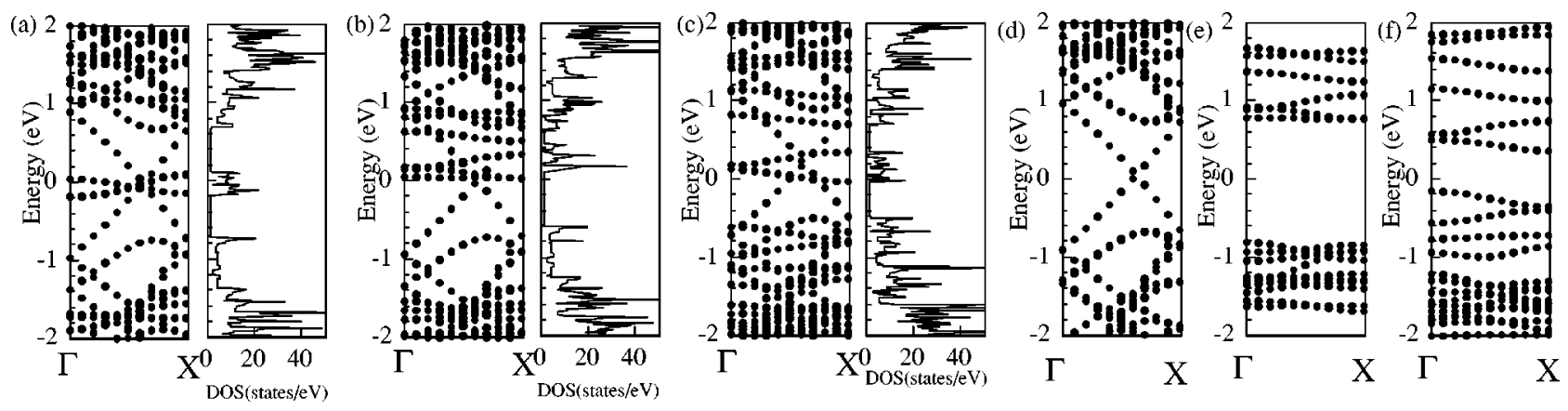

FIG. 5. Energy band structures and density of states of (a) $\mathrm{C}_{60} @(11,11)$, (b) $\mathrm{C}_{70} @(11,11)$, and (c) $\mathrm{C}_{78} @(11,11)$. For comparison, the energy bands of (d) empty $(11,11)$ nanotube, (e) a $\mathrm{C}_{70}$ chain, and (f) a $\mathrm{C}_{78}$ chain are also shown. Energies are measured from the Fermi level energy $E_{F}$. 
the $t_{1 u}$ state of the $\mathrm{C}_{60}$ molecule. Indeed, the electronic structure of the $\mathrm{C}_{78}$ peapod exhibits the multicarrier character: Three energy bands cross $E_{F}$. One of the three states possessing relatively small dispersion corresponds to the lowest conduction band of the $\mathrm{C}_{78}$ chain and the other two have linear dispersion originated from the nanotube [Fig. 5(c)]. Furthermore, the bottom of the conduction band of $\mathrm{C}_{78}$ in the $(11,11)$ nanotube is obviously lower than that of $\mathrm{C}_{60}$ in the nanotube [Figs. 5(a) and 5(c)].

Since the density of states (DOS) elucidates the multicarrier character of the systems, we calculate the DOS of $\mathrm{C}_{60} @(11,11), \mathrm{C}_{70} @(11,11)$, and $\mathrm{C}_{78} @(11,11)$ near $E_{F}$. For integration over the one-dimensional Brillouin zone, we use $20 k$ points in the first Brillouin zone. The calculated DOS of $\mathrm{C}_{78} @(11,11)$ and $\mathrm{C}_{60} @(11,11)$ clearly indicate the multicarrier character in which we find considerably large DOS at $E_{F}\left[N\left(E_{F}\right)\right]$. The estimated values of $N\left(E_{F}\right)$ are 8.1 states/eV/unit and 2.8 states/eV/unit for $\mathrm{C}_{78} @(11,11)$ and the $\mathrm{C}_{60} @(11,11)$, respectively, which are considerably larger than that in the empty carbon nanotubes.

In TEM images, fullerenes are occasionally dimerized in nanotubes. In Fig. 5, the filling of energy bands near $E_{F}$ is fractional and irrational. Hence, a simple Peierls-type metalinsulator transition is unlikely. We thus expect that the multicarrier character may remain even in dimerized peapods. Orientational disorder of fullerenes is also expected. Yet we expect that the electron states of peapods may exhibit similar characteristics to those reported here. This is because the relative orientation, which may cause level splitting, plays a minor role in determining the center-of-mass position of the elecrtron states considered. Positional disorder of fullerenes may cause localization of electron states, although the relative position of electron states is determined by the electron states of an individual fullerene and by the space in the vicinity.

In summary, we have found that the electronic structure of the peapods near the $E_{F}$ depends on the space between encapsulated fullerenes and the nanotube. The hybridization between the NFE states of the nanotubes and the $\pi$ electron states of the fullerenes causes the downward shift of not only LU state but also HO state of the fullerenes. Furthermore, we have also demonstrated that the electron states of peapods reflect those of the encapsulated fullerenes. Thus, the results give the possibility of the Fermi level engineering of the peapods by controlling the space in the tube and the species of the encapsulated fullerenes.

We benefitted from fruitful conversations with $\mathrm{H}$. Kataura and K. Hirahara. This work was partly supported by ACTJST (Japan Science and Technology Corporation), Special Research Project on Nanoscience in University of Tsukuba, NEDO under the Nanotechnology Materials Program, and Grant-in-Aid for Scientific Research from Ministry of Education, Science, and Culture of Japan. Computations were done at Institute for Solid State Physics, University of Tokyo, at Science Information Processing Center, University of Tsukuba, and at Research Center of the Computational Science, Okazaki National Institute.
${ }^{1}$ S. Iijima, Nature (London) 354, 56 (1991).

${ }^{2}$ N. Hamada, S. Sawada, and A. Oshiyama, Phys. Rev. Lett. 68, 1579 (1992).

${ }^{3}$ R. Saito, M. Fujita, M.S. Dresselhaus, and G. Dresselhaus, Appl. Phys. Lett. 60, 2204 (1992).

${ }^{4}$ P.M. Ajayan and S. Iijima, Nature (London) 361, 333 (1993).

${ }^{5}$ R.S. Lee, H.J. Kim, J.E. Fisher, A. Thess, and R.E. Smalley, Nature (London) 388, 255 (1997).

${ }^{6}$ C. Bower, S. Suzuki, K. Tanigaki, and O. Zhou, Appl. Phys. A: Mater. Sci. Process. 67, 47 (1998).

${ }^{7}$ C. Bower, A. Kleinhammes, Y. Wu, and O. Zhou, Chem. Phys. Lett. 288, 481 (1998).

${ }^{8}$ B.W. Smith, M. Monthioux, and D.E. Luzzi, Nature (London) 396, 323 (1998).

${ }^{9}$ B. Burteaux, A. Claye, B.W. Smith, M. Monthioux, D.E. Luzzi, and J.E. Fischer, Chem. Phys. Lett. 310, 21 (1999).

${ }^{10}$ B.W. Smith, M. Monthioux, and D.E. Luzzi, Chem. Phys. Lett. 315, 31 (1999).

${ }^{11}$ J. Sloan, R.E. Dunin-Borkowskib, J.L. Hutchisonb, K.S. Colemana, V.C. Williamsa, J.B. Claridgea, A.P.E. Yorka, C. Xua, S.R. Baileya, G. Browna, S. Friedrichsa, and M.L.H. Green, Chem. Phys. Lett. 316, 191 (2000).

${ }^{12}$ H. Kataura, Y. Kumazawa, Y. Maniwa, I. Umezu, S. Suzuki, Y. Ohtsuka, and Y. Achiba, Synthetic Metals, 121, 1195 (2000).

${ }^{13}$ K. Hirahara, S. Bandow, K. Suenaga, H. Kato, T. Okazaki, H.
Shinohara, and S. Iijima, Phys. Rev. B 64, 115420 (2001).

${ }^{14}$ K. Hirahara, K. Suenaga, S. Bandow, H. Kato, T. Okazaki, H. Shinohara, and S. Iijima, Phys. Rev. Lett. 85, 5384 (2000).

${ }^{15}$ Y. Miyamoto, A. Rubio, X. Blase, M.L. Cohen, and S.G. Louie, Phys. Rev. Lett. 74, 2993 (1995).

${ }^{16}$ S. Okada, A. Oshiyama, and S. Saito, Phys. Rev. B 62, 7634 (2000).

${ }^{17}$ T. Miyake and S. Saito, Phys. Rev. B 65, 165419 (2002).

${ }^{18}$ N.A.W. Holzwarth, S.G. Louie, and S. Rabii, Phys. Rev. B 26, 5382 (1982).

${ }^{19}$ M. Posternak, A. Baldereschi, A.J. Freeman, E. Wimmer, and M. Weinert, Phys. Rev. Lett. 50, 761 (1983).

${ }^{20}$ M. Posternak, A. Baldereschi, A.J. Freeman, and E. Wimmer, Phys. Rev. Lett. 52, 863 (1984).

${ }^{21}$ S. Okada, S. Saito, and A. Oshiyama, Phys. Rev. Lett. 86, 3835 (2001).

${ }^{22}$ P. Hohenberg and W. Kohn, Phys. Rev. 136, B864 (1964).

${ }^{23}$ W. Kohn and L.J. Sham, Phys. Rev. 140, A1133 (1965).

${ }^{24}$ J.P. Perdew and A. Zunger, Phys. Rev. B 23, 5048 (1981).

${ }^{25}$ D.M. Ceperley and B.J. Alder, Phys. Rev. Lett. 45, 566 (1980).

${ }^{26}$ N. Troullier and J.L. Martins, Phys. Rev. B 43, 1993 (1991).

${ }^{27}$ L. Kleinman and D.M. Bylander, Phys. Rev. Lett. 48, 1425 (1982).

${ }^{28}$ O. Sugino and A. Oshiyama, Phys. Rev. Lett. 68, 1858 (1992).

${ }^{29}|\Delta E|$ for $\mathrm{C}_{60} @(10,10)$ is larger than that obtained in our previ- 
ous calculation ${ }^{21}$ in which the geometry of $\mathrm{C}_{60} @(10,10)$ is not completely optimized due to the computational limitation. Despite large difference in $\Delta E$, the difference of each atomic position between present and previous geometries is less than 0.03 A.
${ }^{30} \mathrm{~K}$. Hirahara (unpublished).

${ }^{31}$ K. Kikuchi, N. Nakahara, T. Wakabayashi, S. Suzuki, H. Shiromaru, Y. Miyake, K. Saito, I. Ikemoto, M. Kainosho, and Y. Achiba, Nature (London) 357, 142 (1992). 